\title{
A Review of Existing Transtibial Bionic Prosthesis: Mechanical Design, Actuators and Power Transmission
}

\author{
Ade Reza Ismawan ${ }^{1,2 *}$, Toni Prahasto ${ }^{1}$, Mochammad Ariyanto ${ }^{3}$, Budi Setiyana ${ }^{1}$, Robin \\ Novriansyah ${ }^{4}$ \\ ${ }^{I}$ Mechanical Engineering Department, Diponegoro University, Indonesia \\ ${ }^{2}$ Center for Biomechanics, Biomaterial, Biomechatronics and Biosignal Processing (CBIOM3S), Central Laboratory UNDIP, Semarang, Indonesia \\ ${ }^{3}$ Department of Mechanical Engineering, Graduate School of Engineering, Osaka University, Japan \\ ${ }^{4}$ Department of Orthopaedic and Traumatology, Dr. Kariadi Hospital - Faculty of Medicine Universitas Diponegoro, Semarang, Indonesia \\ Email : aderezaismawan@gmail.com
}

\begin{abstract}
Transtibial and transfemoral amputations are the most common amputations in the world, loss of lower extremity result in impaired function extremities and also body balance. A prosthesis is a medical device designed to replace a specific body part to restore function to a body part lost due to an accident or disease. Most doctors strongly recommend the use of a prosthesis so that patients can return to normal activities after undergoing an amputation. Besides functioning to support beauty, the use of prostheses is also to restore the quality of life of prosthetic users, the issue of metabolic energy consumption when walking is also very important in designing transtibial bionic prosthesis because it involves the comfort of the user transtibial prosthesis. Most of the existing transtibial prosthesis products in Indonesia are conventional passive transtibial foot products, and passive prosthesis users show a limp or asymmetrical gait pattern so that conventional passive prosthesis users experience discomfort when walking in the form of pain in the amputated leg and normal foot, which can cause secondary musculoskeletal injuries such as joint disorders. Passive prostheses cannot generate propulsive force during push-off phase (terminal stance and preswing) of the human gait cycle. The use of passive prostheses can also consume 20-30\% more metabolic energy while walking so that it can cause fatigue for the user. Transtibial bionic prosthesis research is growing, transtibial bionic prosthesis can overcome the weakness of passive prosthesis because it can produce push-off during gait cycle and several researchers have shown that bionic prostheses are capable of mimicking the human gait, as well as improve the performance in a more natural gait and normal walking. This study aims to study the existing transtibial bionic prosthesis by comparing between 6 existing designs of powered ankle or transtibial bionic prosthesis that have been published in several publications. The discussion focuses on the design and mechanical systems, actuators related to the selection of motors and drive mechanisms as well as power transmission from actuators to moving components.
\end{abstract}

Keywords: Transtibial prosthesis, Transtibial bionic prosthesis, Passive prosthesis, Human gait, musculoskeletal injuries, Actuator

Article History: Received 21 November 2021; Received in revised form 27 Dec 2021; Accepted 6 January 2022; Available online: 6 January 2022

DOI: $10.14710 /$ jbiomes.1.2.65-73

\section{INTRODUCTION}

More than 100,000 lower limb amputations occur almost every year in the United States [1] and in China according to national statistics data says there are more than 2 million upper and lower limb amputations [2]. A prosthesis is a medical device to replace certain body parts and is also a solution to help patients restore the function of their limbs after their body parts are seriously injured due to an accident or disease. Although the use of passive transtibial prosthesis has restored the quality of life of amputees so that they can return to their activities, in fact the use of passive prostheses shows some problems, they have several drawbacks when the patient walks with a passive prosthesis showing a limp or asymmetrical gait pattern causing pain in healthy leg and the toe of the amputated leg. In addition, passive prostheses are not able to generate push-off when gait cycle, so when the patient uses a passive prosthesis, the user expends 20$30 \%$ more metabolic energy and will tire more quickly while walking [3].
The use of lower extremity prostheses has increased with the development of the use of carbon fiber for prosthetic legs. The difference between a passive-elastic prosthesis and a biological ankle is that a passive prosthesis can only store and restore thrust without being able to provide propulsive force during plantarflexion [4]. Furthermore, quasi-passive ankle prosthesis using computer-controlled (Össur's Proprio Foot Ankle Prosthesis and élan Ankle Prostheses) and being able to adjust the position during gait cycle has improved better gait stability, however it still unable to imitate normal function of human foot ankle. Patients wearing both passive and quasi-passive prostheses show gait pathology with a greater need for energy metabolism, a limp or asymmetrical gait pattern, and reduced walking speed. Although many causes can be attributed to musculoskeletal injury in prosthesis wearers, a limp or asymmetric gait pattern, the magnitude of the moment at the knee causing pain is the cause of the high risk of musculoskeletal injury in healthy leg and joint degradation [5]. 
Researchers have been undertaking massive research to develop a transtibial bionic prosthesis or active prosthesis over the past few years to correct this deficiency. Active Transtibial prosthesis is divided into two types of active prosthesis, based on the type of joint actuation, the first is the transtibial prosthesis based on pneumatic actuators. In 1998, Klute et al. [6] fabricated a transtibial prosthesis equipped with an artificial pneumatic muscle (Mckibben actuator), and Versluys et al. [7] made another active transtibial leg equipped with an artificial pneumatic cylinder. Although the active transtibial leg which is equipped with pneumatic actuators can produce sufficient propulsive force for the amputee's walking needs, there are difficulties in controlling the active transtibial leg, the inability to adjust the position, and the large dimensions of transtibial prothesis and penumatic size due to the use of a compressed air pump. has kept the development of this pneumatic prosthesis underdeveloped.

The second type of active transtibial is the transtibial prosthesis equipped with a motor drive. According to research with the use of modern active transtibial, the metabolic energy needs of amputees can be reduced by an average of $14 \%$. This active transtibial has a mechanical design that uses a combination of springs and actuators driven by a motor. The position of the spring that is placed parallel to the ankle joint causes the ankle to be more elastic and is useful in restoring the initial position of the sole of the foot during dorsiflexion. The SPARKy active prosthesis is an example of a prosthesis that uses an actuator and is driven by a motor (150-W dc motor) [8]. Another example is the two types of Active Prosthesis that have been made by BiOM [9] and also Spring Active [10]. Recently, a new concept for an energy-efficient transtibial prosthesis was proposed by Cherelle et al. [11], this powered active prosthesis is equipped with a spring so that it can store energy at initial and middle and then generate that energy during terminal stance and preswing phase using a lowpower actuator.

There are several things that should be taken into consideration in designing a good active prosthesis: (1) able to imitate the natural motion of the human ankle joint and can move dorsiflexion and plantarflexion as well as generate propulsive force during the walking gait cycle; (2) low energy consumption; and (3) the weight of the transtibial prosthesis must not exceed the weight of the lost leg.

The weight of the active transtibial prosthesis is an important concern in the design of modern prostheses. Today's modern active transtibial legs are typically equipped with parallel springs, high-power motors and large capacity batteries, focused on generating propulsion during gait cycle and longer use of the transtibial leg and proven to improve energy efficiency, but in fact they have the disadvantage that the weight of the prosthetic transtibial leg can be more than $2 \mathrm{~kg}$, a heavy transtibial leg has the detrimental effect of causing injury and pain in the healthy leg and the stumps of the amputated leg. The lack of a heavy prosthesis can also lead to an inability to provide additional torque, an inability to adapt to variations in terrain, leading to gait asymmetry and gait instability.

\section{Existing Transtibial Bionic Prosthesis}

The currently developed transtibial bionic prosthesis uses actuators and motors to better mimic the function of the human ankle, moving in dorsiflexion and plantarflexion like the human ankle, this bionic transtibial prosthesis can generate propulsive forces due to the push-off generated during the terminal stance and preswing phases [12]. These bionic prostheses generally equiped of a ball screw in series, carbon composite spring, a series elastic actuator and a brushless motor to release and store the motor energy, thereby increasing efficiency. Today's advanced bionic transtibial foot has a foot made from carbon composite making it light and strong, equipped with electronic devices packed in one container, using modular Li-po battery supplies energy to electronic devices and motors and is housed in an external compartment. The weight of the modern transtibial prosthesis is currently estimated at $2.0 \mathrm{~kg}$, resembling the weight of the human ankle joint. This transtibial bionic prosthesis uses feedback data from the prosthetic ankle torsion sensor to ensure that it behaves and resembles the biomimetic function of the ankle constantly by varying the torque and actuator impedance during the gait cycle to match the natural human gait. The control scheme that governs the behavior of the device works naturally, allowing for precise control of timing and ankle strength for various walking speeds. Feedback reflex control of the torsion is used to control transtibial bionic prosthesis, using sensory information from actuator torque at the ankle joint transtibial bionic prosthesis so that the ankle can move in dorsiflexion and plantarflexion more naturally.

For transtibial bionic prosthesis, the actuator functions to transmit the rotational motion of the motor into translational motion so as to produce propulsive force and propel the prosthetic user's body forward during normal walking gait, when designing of transtibaial bionic prosthesis, there are any three important things must be considered: power ,control and actuator. First, so that the transtibial bionic prosthesis can move in dorsiflexion and plantarflexion, an electric motor is needed. To be able to move, it requires power using a commercial battery (eg, lithium-polymer battery), In order for the transtibial bionic prosthesis to be used for at least one full day, In order to be able to control the transtibial bionic prosthesis to adjust movement during the walking phase, Herr et al. [13] suggested that the control used was based on a neuromuscular model. To make the prosthesis adapt to variations in speed and terrain.

The addition of elastic components (eg springs) to the actuators of the transtibial bionic prosthesis serves to mimic the muscle biomechanics of the normal human ankle. When the ankle moves in dorsiflexion (CD), the ankle produces negative work that can be stored by the spring, then when the transtibial bionic prosthesis moves in plantarflexion during the push-off (PP) phase it produces a propulsive force so that the work is positive. The energy generated from the spring during dorsiflexion (CD) is useful to help the actuator work to produce thrust so that the prosthesis user is pushed forward. The combination between a spring or an elastic spring and an actuator is generally called a prosthesis series 
elastic actuator (SEA), parallel elastic actuator (PEA), and SEA + PEA [14].

An example of the Transtibial Bionic Prosthesis developed by Foot-Ankle Prosthesis BiOM at figure 1, the transtibial leg of this bionic prosthesis differs from the transtibial prosthesis of the passive prosthesis and the transtibial leg of the conventional quasi-passive prosthesis to produce positive work in the form of propulsive force during the plantarflexion when push-off phase which can adjust to the walking speed of the user of the transtibial prosthesis. BiOM's transtibial bionic prosthesis uses passive elements and motor driven to mimic a human ankle joint. In Figure 1 shows the mechanical design of the $\mathrm{BiOM}$ foot that mimics the anatomy of the human ankle joint, this transtibial bionic prosthesis is equipped with a carbon series spring, foot made of carbon composite, and an actuator consisting of a motor, timing belt, ball screw, and ball nut so that can produce work positive during the push-off phase of plantar flexion and negative work during dorsiflexion during the gait cycle [15]. This bionic transtibial leg uses a brushless DC motor combined with a series-elastic actuator (SEA), uses a ball screw transmission, and leaf springs made of carbon composite material that are made in series to be able to hold and generate energy when moving in dorsiflexion thereby increasing efficiency, as well as packaged electronic equipment in one compartment. BiOM's transtibial bionic foot uses a carbon composite foot, as well as Modular Lithium-Polymer batteries to supply motor power requirements. The total weight of the Transtibial Bionic Prosthesis developed by BiOM weighs $2.36 \mathrm{~kg}$.
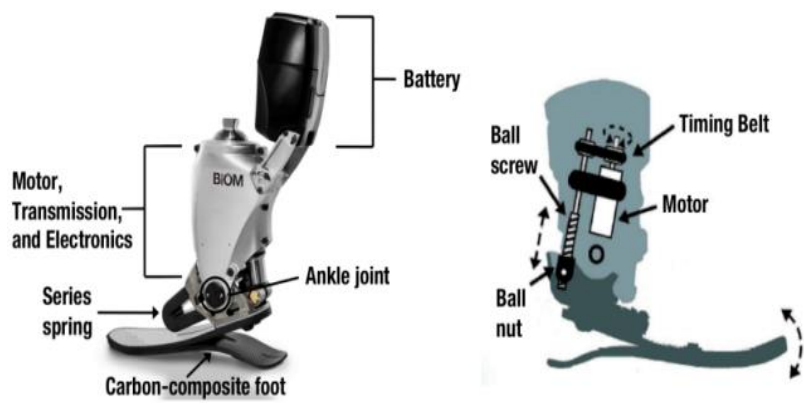

Fig 1. BiOM Foot-Ankle Prosthesis equipped with carbon springs, brushless motor, and carbon composite foot [17]

The feedback data from the sensors is used to move the transtibial bionic foot and mimic the biomimetic function of the ankle, the data is used to adjust the torque requirements during the gait cycle to match the movement of the human foot. This bionic transtibial has a control that mimics biological motion and regulates the speed of the motor to produce the right torque and movement at the right time [16]. Movement scheme settings are carried out via a bluetooth connection between the prosthesis and the android tablet. There are three control parameter schemes used to move the ankle, the first is controlling plantarflexion, time required, and stiffness of the transtibial foot during the walking gait cycle until the desired comfort condition is achieved.

\section{WALKING GAIT CYCLE AND BIOMECHANICS OF HUMAN ANKLE}

Walking is a cycle that starts at the heel strike and ends with the next heel strike on the same foot, there are 2 phases in the walking cycle, namely the stance phase and the swing phase. stance phase which is $60 \%$ of the walking gait cycle starting from heel strike, foot flat, mid stance, heel off and toe off. While the swing phase which is $40 \%$ of the walking gait cycle starting from the toe off, swing and ending with a heel strike. There are three sub-phases in the human walking cycle, namely: $\mathrm{CP}, \mathrm{CD}$, and PP.

a. CP: controlled plantarflexion occurs during heelstrike and ends in foot-flat. In this phase the human ankle produces little torque.

b. CD: controlled dorsiflexion occurs during midstance to before the terminal stance, in that phase the ankle need maximum dorsiflexion and the ankle functions as a spring producing negative work.

c. PP: push-off phase occurs when the terminal stance plantarflexes to preswing, in this push-off phase a large torque is required to produce propulsive force. and in this phase the ankle produces positive work.

So when designing the transtibial bionic prosthesis, the important things is needed such as a spring, control system, and actuator in order to imitate the motion of the human ankle which produces positive work in the form of propulsive force and produces negative work during dorsiflexion.

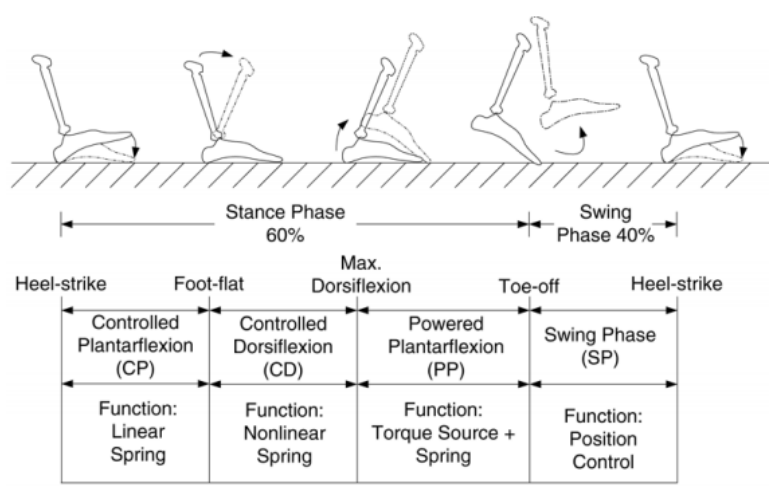

(a)

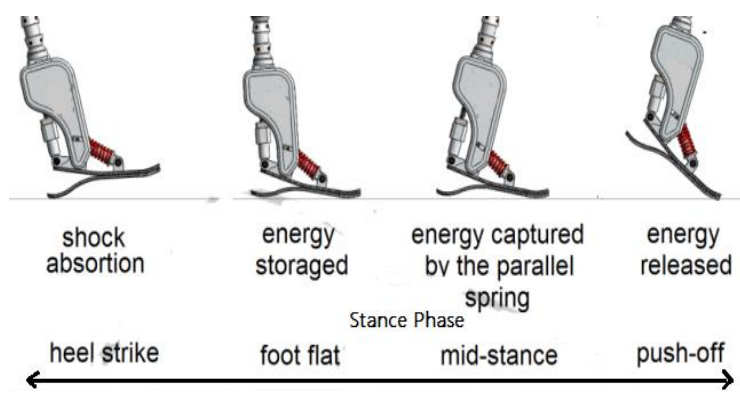

(b)

Fig 2. (a) Walking phases described by normal gait ankle and [15] (b) prosthesis during stance phase [18] 
Table 1 A comparison of powered ankle or transtibial bionic prosthesis: design,actuator and power transmission

\begin{tabular}{|c|c|c|c|c|}
\hline No. & Mechanical Design of Ankle Bionic Prosthesis & Motor driven & Transmission & Advantages \\
\hline 1. & The powered polycentric ankle, Utah University [22] & $\begin{array}{l}\text { - linear actuator with a DC brushless } \\
\text { motor using Maxon Motors, EC } \\
\text { fourpole } 30,200 \mathrm{~W}\end{array}$ & $\begin{array}{l}\text { - using helical-gear } \\
\text { transmission, with } 45^{\circ} \\
\text { helix, and teeth ratio of } \\
\text { 36:12) } \\
\text { - use transmission roller } \\
\text { screw, (Rollvis, lead } 2 \mathrm{~mm} \text {, } \\
\text { with a } 90 \% \text { efficiency) }\end{array}$ & $\begin{array}{l}\text { - total Weight ; } 1,32 \mathrm{~kg} \\
\text { - Range of Motion (RoM) } \\
\text { of this powered } \\
\text { polycentric is } 28^{\circ} \text { for } \\
\text { dorsiflexion to } 27^{\circ} \text { in } \\
\text { plantarflexion. }\end{array}$ \\
\hline 2. & $\begin{array}{l}\text { BiOM powered ankle-foot prosthesis, MIT Cambridge, } \\
\text { Massachusetts, USA [17] }\end{array}$ & $\begin{array}{l}\text { - the transtibial bionic prosthesis uses } \\
\text { SEA combined with brushless DC } \\
\text { motor } 200 \text { Watt } \\
\text { - using modular Li-Po battery }\end{array}$ & $\begin{array}{l}\text { - this transtibial leg is } \\
\text { equipped with ball } \\
\text { screw,timing belt, ball nut } \\
\text { and a carbon-composite } \\
\text { series leaf spring }\end{array}$ & $\begin{array}{l}\text { - the total weight of this } \\
\text { transtibial leg is } 2.36 \mathrm{~kg} \text {. }\end{array}$ \\
\hline
\end{tabular}


3. iWalk BiOM powered ankle-foot prosthesis, MIT Group [23]

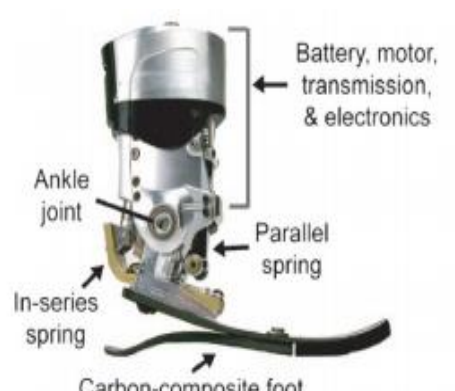

use series-elastic actuator (SEA) combined with DC brushless motor

use belt drive transmission

total prosthesis weigh 200 Watt with reduction

$1.8 \mathrm{~kg}$.

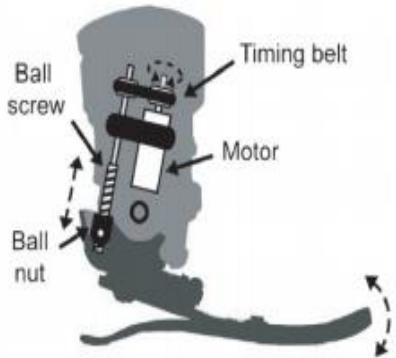

modular Lithium-Polymer battery

$0.22 \mathrm{~kg}$

rque that can be produced

by motor, estimated until 340

$\mathrm{Nm}$

4. Transtibial Bionic Prosthesis from The Chinese University, Hongkong China [24]

- use actuator equipped with

use timing-belt and ball-

118 W DC Maxon motor RE-

screw transmission
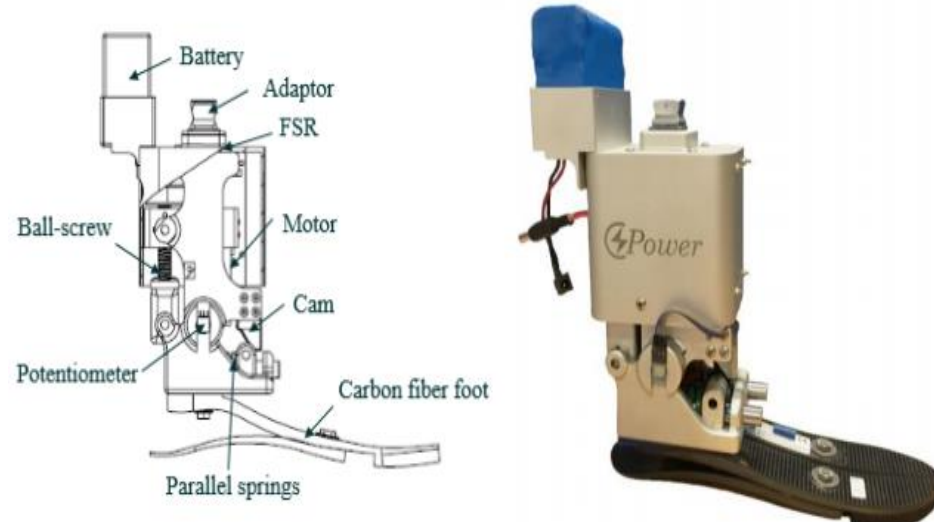

35 and Maxon motor RE-40,

can produce maximum torque

until $120 \mathrm{Nm}$ 
5. Walk-Run ankle, Ruggedized Odyssey Ankle (ROA) Technische Universität, Darmstadt Germany [25]

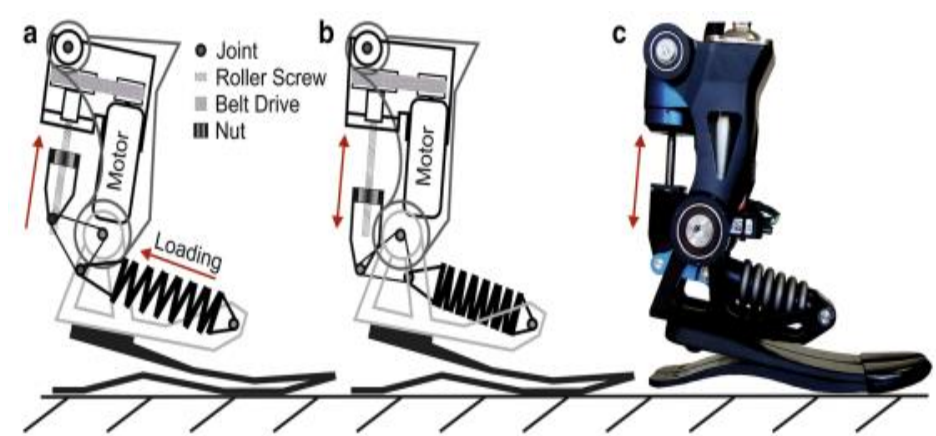

- customized actuator use brushless DC motor $200 \mathrm{~W}$

- for control use PC-104 and battery $4400 \mathrm{mAh}$, produce maximum ankle torque 190 $\mathrm{Nm}$.

\section{transmission use roller} screw and belt drive
- weight of prosthesis is

$1.9 \mathrm{~kg}$, without battery

and the electronics

device and total weight

$2.4 \mathrm{~kg}$

- Range of Motion

(RoM),

$26^{\circ}$ in dorsiflexion, $38^{\circ}$

in plantarflexion.
6. Active Transtibial Bionic Prosthesis, Peking University, Beijing, China [26]
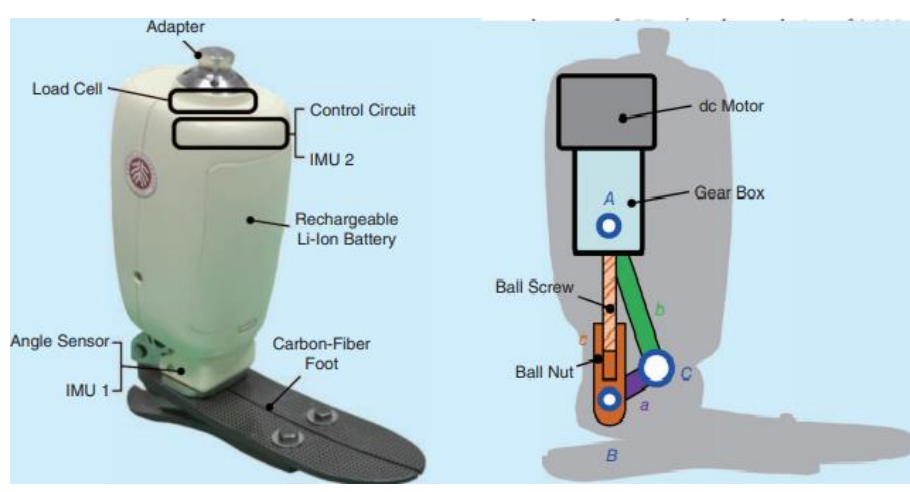

uses a 50W DC brushless motor, Maxon EC $4550 \mathrm{~W}$, and generated to reduction gearbox use transmission ball screw
ROM of ankle bionic foot, $25^{\circ}$ plantar flexion and $25^{\circ}$ dorsiflexion

- the total weight of prosthesis $1.3 \mathrm{~kg}$ without Li-ion battery 


\section{COMPARISON OF TRANSTIBIAL BIONIC PROSTHESIS}

This paper discusses a comparison between 6 existing designs of powered ankle or transtibial bionic prosthesis which have been published in several publications. The discussion focuses on (i) design and mechanical systems, (ii) actuators related to the selection of motors and drive mechanisms and (iii) power transmission from actuators to moving components.

In Table 1, the prostheses compared in this paper are: (i) Polycentric powered ankle, University of Utah, USA (ii) BiOM powered ankle prosthesis, MIT Cambridge, Massachusetts, USA, (iii) iWalk BiOM powered ankle-foot prosthesis, Biomechatronics from MIT, (iv) Powered AnkleFoot Prosthesis equiped parallel spring from The Chinese University of Hongkong, China and (v) Walk-Run ankle, Ruggedized Odyssey Ankle (ROA) Technische Universität, Darmstadt Germany, and (vi) Active Transtibial Prosthesis, Research Group from Peking University.

This study is useful in developing the design of the bionic foot in the future, the selection of linear actuators and the type of transmission are important considerations to produce the required torque. In this study, a linear actuator has not been determined which is recommended as a bionic foot drive in order to perform plantarflexion and dorsiflexion movements. At the previous study of the bionic hand by researchers of CBIOM3S [19-21], the use of a linear actuator acts as the main driven in moving the fingers, this is an opportunity for research on the use of linear actuators on transtibial bionic prosthesis to be open.

\section{Conclusion}

Issue energy efficiency is imprortant, developed a Transtibial Bionic Prosthesis can produce prospulsive force for positive work and can reduce metabolic energy consumption. In the design of Transtibial Bionic Prosthesis, there are three issues should be considered: actuator, power and control. Firstly. Based on comparison, BiOM powered ankle-foot prosthesis, iWalk BiOM transtibial bionic prosthesis, Walk-Run ankle of Ruggedized Odyssey Ankle (ROA) using linear actuators equipped with springs can provide positive net power to the ankle during the standing phase while walking gait cycle, some of the benefits are absorbing and providing impulse forces when walking like the human biological foot, the use of linear actuators and springs also serves to increase safety and stability to avoid assymetric gait pattern, pain at the stump, and injuries at healthy leg. Selection of the appropriate linear actuator has an impact on the torque generated, the torque and lightweight like design of Lightweight Active Transtibial Prosthesis by Peking University of the transtibial bionic prosthesis have an effect on the consumption of metabolic energy, which has an impact on comfort so that users don't tire quickly when using the powered ankle. Based on comparisons, the authors recommend the design Walk-Run ankle foot to be further developed because it can be used for walking and running at a medium speed, but the consideration of torque and weight is an important concern in the design of the transtibial bionic prosthesis, because the bionic foot is light and the appropriate torque has an impact on metabolic energy consumption and stability.

\section{ACKNOWLEDGMENTS}

The authors acknowledge to LPPM Diponegoro University for providing the research fund for this research based on the contract number: 233-131/UN7.6.1/PP/2020.

\section{REFERENCES}

[1] Uustal H, 2006. Prosthetics and Orthotics, in Essential Physical Medicine and Rehabilitation, G. Cooper, Ed. Humana Press, pp. 101-118

[2] China Disabled Persons Development Statistics Bulletin, Chinese Disabled Persons' Federation, Beijing, China

[3] Molen NH, 1973. Energy/speed relation of below-knee amputees walking on motor-driven treadmill," Int. Z. Angew. Physiol., vol. 31, no. 3, pp. 173-185

[4] Bateni H, Olney SJ, 2002. Kinematic and kinetic variations of below-knee amputee gait. Prosthetic and Orthotic Science, 14:2-10

[5] Sanderson DJ, Martin PE, 1997. Lower extremity kinematic and kinetic adaptations in unilateral belowknee amputees during walking. Gait Posture 1997, 6:126-136.

[6] Klute GK, Czerniecki J, and Hannaford B, 1998. Development of powered prosthetic lower limb, in Proc. 1st Nat. Meeting, Veterans Affairs Rehabil. R and D Service, Washington, DC

[7] Versluys R, et al, 2008. A biomechatronical transtibial prosthesis powered by pleated pneumatic artificial muscles, Int. J. Model. Identif. Control, vol. 4, no. 4, pp. 394-405

[8] Hitt J, et al, 2009. Robotic transtibial prosthesis with biomechanical energy regeneration, Ind. Robot, vol. 36 , no. 5, pp. 441-447

[9] (2014, Feb. 22). BiOM Personal Bionics. [Online]. Available: http://www. biom.com/

[10] SpringActive. (2014, Feb. 22). Providing innovative solutions to powered human assistance. [Online]. Available: http://www.springactive.com/

[11] Cherelle P, et al, 2014. Design and validation of the ankle mimicking prosthetic (AMP-) foot 2.0, IEEE Trans. Neural Syst. Rehabil. Eng., vol. 22, no. 1, pp. 138-148

[12] Au S, Herr H, 2008. Powered ankle-foot prosthesis The importance of series and parallel motor elasticity. IEEE Robot Autom Mag, 15:52-59

[13] Eilenberg M F, Geyer H, and Herr H,2010. Control of 
a powered ankle -foot prosthesis based on a neuromuscular model. IEEE Trans. Neural Systems and Rehabilitation Engineering, pp. 164 -173

[14] Au S, Weber J, and Herr H,2009. Powered ankle -foot prosthesis improves walking metabolic economy," IEEE Trans. Robotics, pp. 51 -66

[15] Au S, Berniker M, and Herr H,2008. Powered anklefoot prosthesis to assist level-ground and stair-descent gaits, Neural Networks, vol. 21, no. 4, pp. 654-666

[16] Markowitz J, et al, 2011. Speed adaptation in a powered transtibial prosthesis controlled with a neuromuscular model, Phil. Trans. R. Soc. B, vol. 366, no. 1570, pp. $1621-1631$

[17] Hill D, and Herr H,2013. Effects of a powered anklefoot prosthesis on kinetic loading of the contralateral limb: A case series, IEEE International Conference on Rehabilitation Robotics.

[18] Martinez R, et al,2014. A Low Cost Design of Powered Ankle-Knee Prosthesis for Lower Limb Amputees Preliminary Results.In Proceedings of the International Conference on Biomedical Electronics and Devices - Volume 1: BIODEVICES, (BIOSTEC 2014) ISBN 978-989-758-013-0, pages 253-258. DOI: 10.5220/0004914402530258

[19] Ismail R, Ariyanto M, Hidayat T, Setiawan JD,2019. Design of fabric-based soft robotic glove for hand function assistance. In2019 6th International Conference on Information Technology, Computer and Electrical Engineering (ICITACEE) 2019 Sep 26 (pp. 1-5). IEEE.

[20] Ariyanto M, Ismail R, Setiawan JD, Yuandi EP,2019. Anthropomorphic transradial myoelectric hand using tendon-spring mechanism. TELKOMNIKA. 2019 Feb 1;17(1):537-48.

[21] Ismail R, Wijaya GD, Ariyanto M, Suriyanto A, Caesarendra W,2018. Development of Myoelectric Prosthetic Hand based on Arduino IDE and Visual C\# for Trans-radial Amputee in Indonesia. International
Conference on Applied Engineering (ICAE) 2018 Oct 3 (pp. 1-5). IEEE.

[22] Cempini M, Hargrove LJ, and Lenzi T,2017. Design, Development, and Bench-top Testing of a Powered Polycentric Ankle Prosthesis

[23] Eilenberg MF, Geyer H, and Herr H,2009. Control of a Powered Ankle-Foot Prosthesis Based on a Neuromuscular Model, IEEE Transactions on Neural Systems and Rehabilitation Engineering (TNSRE)

[24] Gao F, Liu Y, and Hsin Liao W,2016. A New Powered Ankle-Foot Prosthesis with Compact Parallel Spring Mechanism, Proceedings of the 2016 IEEE International Conference on Robotics and Biomimetics Qingdao, China, December 3-7, 2016.

[25] Grimmer et al, 2016. A powered prosthetic ankle joint for walking and running, BioMed Eng OnLine, 15(Suppl 3):141, Berlin, Germany

[26] Wang Q,et al,2015. Walk the Walk: A Lightweight Active Transtibial Prothesis, IEEE Robotics \& Automation Magazine 